\section{Experiencia inicial de radioterapia estereotáxica (SBRT) en metástasis óseas de columna vertebral}

\author{
NATALIA JARA O. ${ }^{\text {, }}$, CLAUDIO SOLÉ P. ${ }^{1,2}$, SEBASTIÁN SOLÉ Z. ${ }^{1,2}$
}

\section{Stereotactic body radiation therapy (SBRT) for pain management in spine bone metastases}

Background: Treatment of spine bone metastases with stereotactic radiotherapy (SBRT) may produce greater pain relief than palliative radiotherapy. Aim: To evaluate the analgesic response to SBRT. Material and Methods: A search was made in an electronic database of all patients treated by SBRT in our center. We found 20 patients that were treated with SBRT in the spine on 24 sites (lesions). Analgesic response was evaluated at 3 and 6 months after SBRT. Other factors such as age, sex, functional status, diagnosis, metastasis location, dosimetry and toxicity of the treatment were also described. Results: The median follow-up was 8.1 months. Complete pain reliefoccurred at three months in 74\% of the treated sites. At three months, $78 \%$ of the patients presented a functional status 0 (ECOG). The median dose used was $24 \mathrm{~Gy}$ in 2 fractions. No cases of $G 3$ or greater toxicity were recorded. Conclusions: The analgesic response to $S B R T$ seems to be better than that reported for palliative radiotherapy.

(Rev Med Chile 2019; 147: 993-996)

Key words: Neoplasm Metastasis; Radiosurgery; Spine.

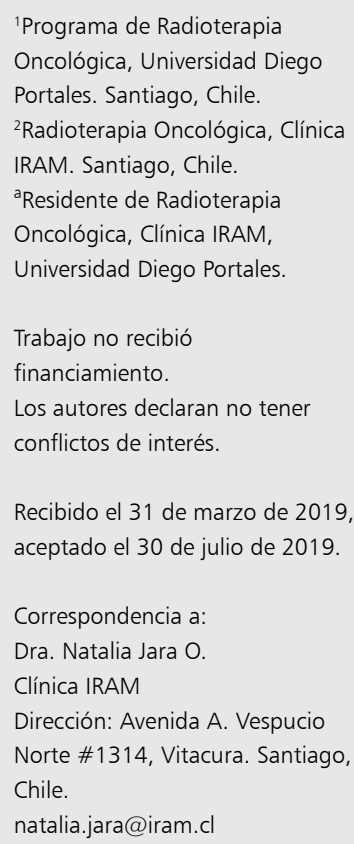

Recibido el 31 de marzo de 2019 aceptado el 30 de julio de 2019.

Correspondencia a:

Dra. Natalia Jara O.

Clínica IRAM

Dirección: Avenida A. Vespucio Norte \#1314, Vitacura. Santiago, Chile.

natalia.jara@iram.cl

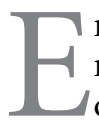
n Chile cada año se diagnostican 52.500 nuevos casos de cáncer ${ }^{1}$. Se estima que $30 \%$ de estos pacientes desarrollan metástasis óseas, de los cuales dos tercios se localizan en la columna vertebral, siendo la región torácica y lumbar los sitios más frecuentes ${ }^{2}$. Estas lesiones pueden producir dolor y otros síntomas que afectan la calidad de vida de los pacientes y su estado funcional.

Los tratamientos de radioterapia paliativa que usan dosis bajas de radiación tienen resultados aceptables en el control del dolor, que van en un rango de $23 \%$ hasta $60 \%$ según diferentes revisiones sistemáticas ${ }^{3-5}$. La duración de estos tratamientos puede variar entre 1 a 10 sesiones $(0,14$ a 2 semanas de tratamiento).

Aumentar la dosis de radiación por sesión podría lograr mejorar el control del dolor sin aumentar el número de sesiones. Pero con las técnicas convencionales de radiación no es posible otorgar una dosis más alta sin poner en riesgo órganos sanos adyacentes a la lesión, como, por ejemplo, la médula espinal, el esófago, el intestino, etc. Gracias al desarrollo de nuevas tecnologías en radioterapia se puede subir la dosis por sesión de manera segura. Esta técnica es la radioterapia estereotáxica (SBRT), que permite máxima precisión en la entrega de la dosis con gran gradiente de ella entre el tumor y los órganos cercanos ${ }^{6}$. Varios estudios no randomizados sugieren un mejor control del dolor utilizando SBRT versus lo reportado con radioterapia paliativa ${ }^{7-9}$.

El objetivo de este trabajo es revisar el resultado en control del dolor en nuestros pacientes sometidos a SBRT por metástasis óseas en la columna vertebral. 


\section{Pacientes y Métodos}

\section{Sujetos y objetivos}

Estudio descriptivo, retrospectivo donde se revisaron las fichas de pacientes con metástasis óseas en la columna vertebral tratados con SBRT desde el 1 de diciembre del 2016 al 30 de agosto 2018 en Clínica IRAM. A todos los pacientes se les indicó SBRT debido a que tenían oligometástasis (5 o menos lesiones) con tumor primario controlado. Todos los pacientes evaluados tienen diagnóstico de cáncer con confirmación patológica y consentimiento informado para participar en el estudio.

Se excluyeron del trabajo a aquellos pacientes tratados en escenario de re-irradiación.

Este trabajo fue aceptado por el comité de ética científico adultos del Servicio de Salud Metropolitano Oriente.

El objetivo primario de este estudio fue evaluar los resultados en respuesta analgésica con el tratamiento con SBRT. La respuesta analgésica, se definió como la disminución del dolor en 2 o más puntos en la escala visual análoga (EVA $)^{11}$.

Los objetivos secundarios a evaluar fueron el control local, definido como la no evidencia de progresión ya sea clínica y/o imagenológica. Las características demográficas (edad, sexo, estado funcional según $\mathrm{ECOG}^{10}$ ), los sitios de localización de las metástasis, parámetros de calidad de radiación (dosis recibida en órganos de riesgo, volúmenes de tratamiento) y toxicidad del tratamiento.

La toxicidad fue evaluada según CTCAE (Versión 4.0$)^{13}$.

Todos los parámetros descritos fueron evaluados a los 3 y 6 meses de terminado el tratamiento.

\section{Radioterapia}

La técnica de radioterapia utilizada fue la misma para todos los pacientes.

La simulación se realizo en decúbito supino. Para el posicionamiento, se utilizó mascara termoplástica con hombros para lesiones ubicadas en columna cervical y torácica hasta T4. Para lesiones ubicadas desde T5 hasta el sacro se utilizó vac-lock. Las imágenes para la simulación fueron obtenidas por scanner con cortes de $2 \mathrm{~mm}$ y se realizó una fusión con las imágenes de RM en secuencias T1 y T2.

Para definir los volúmenes de tratamiento se utilizaron las recomendaciones del Consenso Internacional para Radiocirugía ${ }^{14}$.
La dosis prescrita evaluada fue 24 Gy en 2 sesiones y 30 Gy en 5 sesiones. En la dosimetría y planificación se utilizó el programa ECLIPSE. La prescripción del CTV/PTV y los valores de tolerancia de los órganos a riesgo fueron los del protocolo NCIC CTG: SC $24^{15}$ (Tabla 1). Todos los pacientes fueron tratados en un acelerador lineal "Varian Trilogy HD", con técnica IMRT/VMAT, con fotones de $6 \mathrm{MV}$. Se realizó comprobación del posicionamiento con Cone-Beam CT antes de cada sesión de tratamiento.

\section{Resultados}

Se evaluaron 20 pacientes con 24 lesiones. La mediana de seguimiento fue 8,1 meses. Las características demográficas de la población estudiada se describen en la Tabla 2.

El objetivo primario del estudio fue evaluar la respuesta analgésica. $\mathrm{Al}$ inicio del tratamiento la mediana de dolor de los pacientes fue 5 según la escala EVA. Al análisis de los 3 y 6 meses la mediana de dolor fue 0 y 0 según la escala EVA, respectivamente. Los datos de la respuesta analgésica en el tiempo se grafican en la Tabla 3 con su respectiva mediana.

En la Figura 1 se muestra el caso de un paciente que fue tratado por una metástasis de columna lumbar que en el control de los 14 meses muestra osificación casi completa de la lesión y respuesta completa del dolor.

A los 3 meses 89\% (17/19) de los pacientes presentó respuesta analgésica completa y a los 6 meses 100\% (13/13).

Tabla 1. Valores de tolerancia según protocolo NCIC CTG: SC24

\begin{tabular}{|c|c|c|}
\hline Órgano & & $\begin{array}{l}\text { Limite } \\
\text { de dosis }\end{array}$ \\
\hline Médula Espinal y Cauda & Punto máximo dosis & $<17$ Gy \\
\hline Riñones & $\begin{array}{l}\text { Punto máximo dosis } \\
\text { Dosis media }\end{array}$ & $\begin{array}{r}<24 \mathrm{~Gy} \\
<5 \mathrm{~Gy}\end{array}$ \\
\hline $\begin{array}{l}\text { Esófago, Estómago } \\
\text { Intestino, Recto }\end{array}$ & Punto máximo dosis & $<20 \mathrm{~Gy}$ \\
\hline Pulmones & $\begin{array}{l}\text { V10 } \\
\text { V5 } \\
\text { V20 } \\
\text { Dosis media }\end{array}$ & $\begin{array}{r}<10 \% \\
<35 \% \\
<3 \% \\
<5 \%\end{array}$ \\
\hline
\end{tabular}


Tabla 2. Características demográficas

\begin{tabular}{|c|c|c|}
\hline n & 20 pacientes & $\begin{array}{l}24 \text { localiza- } \\
\text { ciones }\end{array}$ \\
\hline $\begin{array}{l}\text { Edad } \\
\text { Mediana, (rango) }\end{array}$ & 54,3 años & $(25-73)$ \\
\hline Género & $\begin{array}{l}60 \% \text { Mujeres } \\
40 \% \text { Hombres }\end{array}$ & \\
\hline Sitio primario & $\begin{array}{l}\text { 40\% Mama } \\
\text { 15\% Próstata } \\
\text { 10\% Pulmón } \\
35 \% \text { Otros }\end{array}$ & \\
\hline $\begin{array}{l}\text { Localización de } \\
\text { metástasis }\end{array}$ & $\begin{array}{l}\text { 50\% Lumbosacro } \\
\text { 46\% Torácica } \\
\text { 4\% Cervical }\end{array}$ & \\
\hline $\begin{array}{l}\text { Mediana de dosis } \\
\text { de tratamiento }\end{array}$ & $24 \mathrm{~Gy} / 2 \mathrm{Fx}$ & $\begin{array}{l}\mathrm{BED}=120 \\
\alpha / \beta: 3\end{array}$ \\
\hline $\begin{array}{l}\text { Seguimiento } \\
\text { Mediana, (rango) }\end{array}$ & 8,1 meses & $(20-3)$ \\
\hline
\end{tabular}

Tabla 3. Resultados de respuesta analgésica y capacidad funcional

\begin{tabular}{|lccc|}
\hline Tiempo & 0 meses & $\mathbf{3}$ meses & $\mathbf{6}$ meses \\
EVA & 5 & 0 & 0 \\
Mediana, (rango) & $(0-10)$ & $(0-4)$ & $(0-4)$ \\
ECOG & 1 & 0 & 0 \\
Mediana, (rango) & $(0-2)$ & $(0-1)$ & $(0-1)$ \\
Número de pacientes & 20 & 19 & 13 \\
\hline
\end{tabular}

Se muestran los datos de respuesta analgésica según escala EVA y la capacidad funcional según ECOG, al inicio del tratamiento y al control de 3 y 6 meses post tratamiento.

$\mathrm{Al}$ inicio del tratamiento $75 \%$ de los pacientes presentaban un estado funcional ECOG 1 . A los 3 meses $78 \%$ presentan ECOG 0 y a los 6 meses $85 \%$ de los pacientes presentan ECOG 0. Los datos del
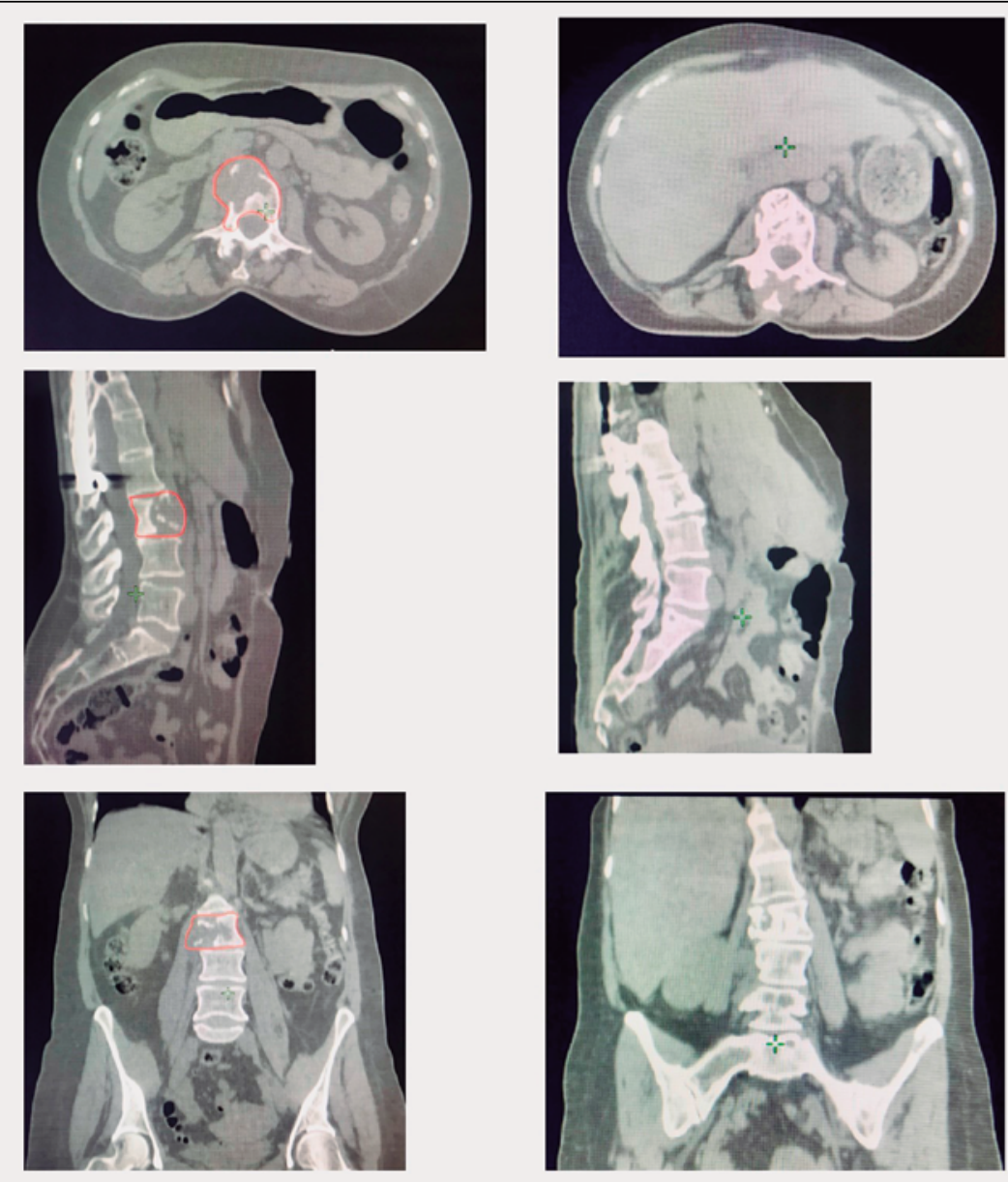
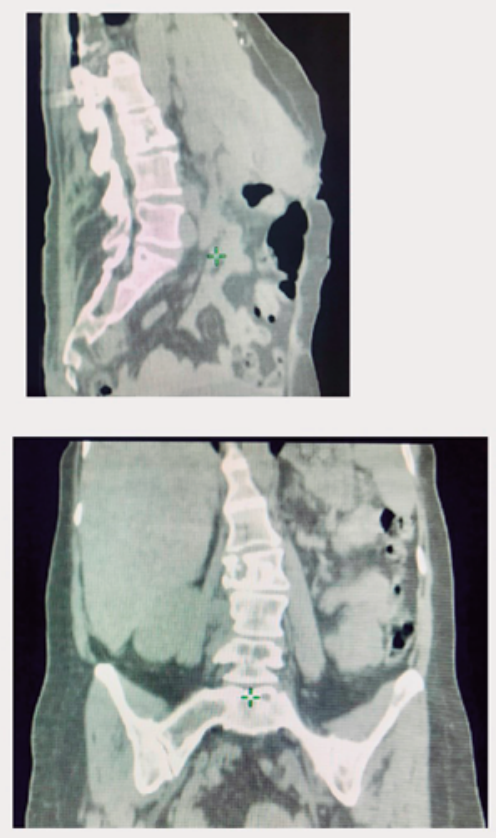

Figura 1. Resultado de tratamiento con SBRT. Se presenta el caso de un paciente a la derecha las imágenes de la lesión al inicio y a la izquierda las imágenes de control a los 14 meses post tratamiento. 
estado funcional en el tiempo se muestran en la Tabla 3 con su respectiva mediana.

En relación a los parámetros dosimétricos la mediana de dosis fue 24 Gy en 2 fracciones, que fue prescrita 17 de los 20 pacientes, a un volumen promedio del PTV fue 105,4 cc.

No hay registro de toxicidad grado 3 o mayor.

\section{Discusión}

Este es el primer trabajo latinoamericano en presentar los resultados con radioterapia estereotáxica para el tratamiento de metástasis en columna vertebral.

Si bien es un estudio descriptivo retrospectivo de una sola institución, los resultados obtenidos en respuesta analgésica son superiores a lo reportado con radioterapia paliativa y son similares a lo descrito por otros grupos con SBRT. Actualmente hay 2 estudios randomizados en curso cuyos resultados aún están pendientes (NCIC SC24 y RTOG 0613).

No hubo reporte de toxicidad severa (grado 3 o mayor) lo que respalda el uso del protocolo del NCIC para evaluar dosis de los órganos de riesgo. Se debe aclarar que la aplicación de esta técnica requiere de tecnología de punta y un equipo entrenado para que se haga de manera segura, ya que existe un potencial mayor riesgo de complicaciones severas si no se hace bien debido a las mayores dosis que son utilizadas.

\section{Referencias}

1. Asociación de Isapres de Chile. Estudio AICH 2017. www.isapre.cl/PDF/Informe\%20Cancer2017.pdf.

2. Sprave T, Verma V, Förster R, Schlampp I, Bruckner T, Bostel $\mathrm{T}$, et al. Randomized phase II trial evaluating pain response in patients with spinal metastases following stereotactic body radiotherapy versus tree-dimensional conformal radiotherapy. Radiother Oncol 2018; 128 (2): 274-82.

3. Chow E, Harris K, Fan G, Tsao M, Sze WM. Palliative radiotherapy trials for bone metastases: a systematic review. J Clin Oncol 2007; 25 (11): 1423-36.

4. Sze WM, Shelley M, Held I, Mason M. Palliation of metastatic bone pain: single fraction versus multifraction radiotherapy - a systematic review of the randomised trials. Cochrane Data base Syst Rev 2004; 2. CD004721.

5. Wu JS, Wong R, Johnston M, Bezjak A, Whelan T. Meta-analysis of dose- fractionation radiotherapy trials for the palliation of painful bone metastases. Int J Radiat Oncol Biol Phys 2003; 55: 594-605.

6. Sahgal A, Weinberg V, Ma L, Chang E, Chao S, Muacevic $A$, et al. Probabilities of radiation myelopathy specific to stereotactic body radiation therapy to guide safe practice. International Journal of Radiation Oncology biology physics 2013; 85: 341-7.

7. Gerszten PC. The Role of Minimally Invasive Techniques in the Management of Spine Tumors: Percutaneous Bone Cement Augmentation, Radiosurgery, and Microendoscopic Approaches. Orthop Clin North Am 2007; 38: 441-50.

8. Chang BK, Timmerman R. Stereotactic Body Radiation Therapy A Comprehensive Review. Am J Clin Oncol 2007; 30: 637-44.

9. Ryu S, Rock J, Rosenblum M, Kim JH. Patterns of failure after single-dose radiosurgery for spinal metástasis. J Neurosurg 2004; 101: 402-5.

10. Oken MM, Creech RH, Tormey DC, Horton J, Davis TE, McFadden ET, et al. Toxicity And Response Criteria Of The Eastern Cooperative Oncology Group. Am J Clin Oncol 1982; 5: 649-55.

11. Bugedo G, Dagnino J, Muñoz H, Torregrosa S. Escala visual análoga: Comparación de seis escalas distintas. Rev Chil Anest 1989; 18: 132.

12. Chow E, Hoskin P, Mitera G, Zeng L, Lutz S, Roos D, et al. Update of the international consensus on palliative radiotherapy endpoints for future clinical trials in bone metastases. Int J Radiat Oncol Biol Phys 2012; 82 (5): 1730-7.

13. Institute NC. Common Terminology Criteria for Adverse Events (CTCAE) Version 4.0. 2009.

14. Cox BW, Spratt DE, Lovelock M, Bilsky MH, Lis E, Ryu $S$, et al. International Spine Radiosurgery Consortium consensus guidelines for target volume definition in spinal stereotactic radiosurgery. Int J Radiat Oncol Biol Phys 2012; 83 (5): 597-605.

15. Sahgal A. A Phase II Randomized Feasibility Study Comparing Stereotactic Body Radiotherapy (SBRT) Versus Conventional Palliative Radiotherapy (CRT) for Patients With Spinal Metastases. NCIC CTG Protocol number: SC.24. 